\title{
Heterogeneous Adaptive Behavioral Responses May Increase Epidemic Burden
}

Baltazar Espinoza ( $\square$ baltazar.espinoza@virginia.edu )

University of Virginia

\section{Samarth Swarup}

University of Virginia

Christopher Barrett

University of Virginia

Madhav Marathe

University of Virginia

\section{Research Article}

Keywords:

Posted Date: January 28th, 2022

DOI: https://doi.org/10.21203/rs.3.rs-1229867/v1

License: (c) (1) This work is licensed under a Creative Commons Attribution 4.0 International License. Read Full License 


\title{
Heterogeneous Adaptive Behavioral Responses May Increase Epidemic Burden
}

\author{
Baltazar Espinoza ${ }^{1, *}$, Samarth Swarup ${ }^{1}$, Christopher L. Barrett ${ }^{1}$, and Madhav Marathe ${ }^{1}$ \\ ${ }^{1}$ Biocomplexity Institute and Initiative, Network Systems Science and Advanced Computing Division, University of \\ Virginia, Virginia, USA \\ *baltazar.espinoza@virginia.edu
}

\begin{abstract}
Non-pharmaceutical interventions (NPIs) constitute the front-line responses against epidemics. Yet, the interdependence of control measures and individuals' microeconomics, beliefs, perceptions and health incentives, is not well understood. Epidemics constitute complex adaptive systems where individual behavioral decisions drive and are driven by, among other things, the risk of infection. To study the impact of heterogeneous behavioral responses on the epidemic burden, we formulate a two risk-groups mathematical model that incorporates individuals' behavioral decisions driven by risk perceptions. Our results show a trade-off between the efforts to avoid infection by the risk-evader population, and the proportion of risk-taker individuals with relaxed infection risk perceptions. We show that, in a structured population, privately computed optimal behavioral responses may lead to an increase in the final size of the epidemic, when compared to the homogeneous behavior scenario. Moreover, we find that uncertain information on the individuals' true health state may lead to worse epidemic outcomes, ultimately depending on the population's risk-group composition. Finally, we find there is a set of specific optimal planning horizons minimizing the final epidemic size, where these depend on the population structure.
\end{abstract}

\section{Introduction}

Non-pharmaceutical interventions (NPIs) constitute a suite of front-line behavioral responses whose collective compliance may assemble a behavioral immune system at the population scale ${ }^{1,2}$. Classical mathematical models use a variety of modeling frameworks to study the interplay between disease dynamics and epidemic mitigation policies ${ }^{3-5}$. However, control policies have historically focused on the population-level consequences, neglecting individual-level incentives and costs associated with complying with public health recommendations ${ }^{6,7}$. Characterization of the effectiveness of control policies and their viability requires understanding how behavioral modifications intended to reshape epidemic dynamics at the population scale interact with individual microeconomics, beliefs, perceptions and health incentives ${ }^{8-11}$. Heterogeneous living conditions (socioeconomic characteristics, beliefs, education, demography, etc.) modulate the behavioral choices individuals make during the epidemic period. Particularly, these impact people's adherence to recommended control policies ${ }^{12,13}$. Consequently, the extent to which control policies are effective in ameliorating the epidemic burden inherently depends on the affected population's structure and the associated behavioral responses ${ }^{14,15}$.

Diverse modeling frameworks have been used to characterize the intertwined dynamics generated by the disease dynamics and the set of behavioral responses available to mitigate disease spread ${ }^{3-5}$. However, most of the mathematical models do not consider the adaptive nature of the co-evolving epidemic and adaptive behavioral dynamics. Usually, behavioral models in epidemiology look only at the current and past system's state, and use that information to infer individuals' behavioral responses. Our behavioral model takes into account the behavior of individuals based on their estimate of the epidemic dynamics at the current state and in the future. During an epidemic, the feedback loop between people's activities to avoid risk of infection and the desire to maintain social interactions and economic productivity creates a complex adaptive system, in which behavioral responses both drive and are driven by the disease transmission process. Recently, the modeling framework proposed by Fenichel et al. ${ }^{16-19}$ envisions individual behavioral adaptations as a Markov decision process by using an epidemiological-economic model. Markov decision processes have found applications and provided foundations in AI for modeling sequential decision-making in diverse contexts ${ }^{20-22}$. In this work we focus on a behavioral aspect extensively documented during the COVID-19 pandemic on different regions, the population's heterogeneous behavioral responses to the risk of infection. We aim to study the impact of heterogeneous behavioral responses on the progression of an epidemic. We extend the epidemiological-economic framework by considering a risk-structured population and information uncertainty. We use a set of ordinary differential equations to model disease progression, and a decentralized Markov decision framework to model the strategic behavior of individuals across risk groups and over different health classes. We assume economic productivity depends on social interactions ${ }^{23,24}$, and model behavioral changes as adjustments in the contact decisions made by 
individuals seeking to maximize the net benefits offered by contacts with others, where these also carry a risk of infection. Beyond our explicit formulation of behavioral responses, our results are robust to other types of behavioral responses that ultimately reduce the risk of infection. We incorporate important features of the COVID-19 pandemic: $(i)$ a large proportion of infected individuals are asymptomatic or show mild symptoms that allow them to continue social interaction, thus becoming major drivers of transmission ${ }^{25-27}$; (ii) the relative infectiousness of exposed and asymptomatic infectious individuals is uncertain $^{28-30}$; and (iii) heterogeneous population behavioral responses driven by differential risk perceptions. The role of non-symptomatic (exposed and asymptomatic) but infectious individuals in our behavioral model formulation is critical. Most social interactions require immediate evaluation of the infection risk, which is assumed to be determined by vulnerability cues ${ }^{31}$. Consequently, in the absence of symptoms, exposed and asymptomatic individuals may both behave and be treated by others as if they are uninfected (i.e., in the susceptible state w.r.t. the epidemic dynamics).

We extend the behavioral modeling framework by Fenichel et al. ${ }^{16-19}$ by: (i) coupling a set of Markov decision processes (MDPs) to incorporate differential adaptive behavioral responses; (ii) using a more complex epidemic model of disease transmission; and (iii) including the role of uncertain information about individual health states, affecting the decision-making processes. Individuals are divided into two classes based on their risk-acceptance behavior. These extensions aim to incorporate pervasive experiences from the ongoing COVID-19 pandemic. Our simulations yield the following insights: (i) there is a balance between the reduction of cases due to risk-evaders' effort to decrease their infection likelihood, and the proportion of the population acting as risk-takers; (ii) the risk-reduction and risk-takers trade-off has the potential to increase the attack rate (the proportion of the population infected over the epidemic), and it is sensitive to the proportion of asymptomatic cases and their relative infectiousness; and (iii) there is a set of optimal planning horizons that minimize the attack rate. The optimal planning horizons depend on the population's structure and the group-specific risk sensitivities. Finally, to the best of our knowledge, this is the first time that multiple MDPs are coupled to study heterogeneous adaptive behavioral responses in an epidemic model.

\section{Results}

Since the proposed behavior model is not amenable to an analytical solution, we numerically explore the implications of adaptive behavior and uncertain information on the epidemic dynamics. We use the final epidemic size as a metric to assess the impact of behavioral responses on different behavioral and epidemiological scenarios. In the absence of appropriate behavioral data, we assume that individuals make an average of $b=50$ contacts per day. Future utility is discounted at the rate of $5 \%$ per year $(\delta=0.99986)$, and the utility function parameter value is assumed to be $v=0.1^{16,18}$. In the Supplementary Information we show that variations on the daily average contacts $(b)$, and the discount rate $(\delta)$ parameters, modify the sensitivity of the behavioral response, but do not change our qualitative results. Given that the early phase of the epidemic is mainly driven in the absence of behavioral responses, we calibrate the behavior model by making the constant contacts model's basic reproductive number $\left(\mathscr{R}_{0}\right)$ to be consistent with early disease dynamics of the COVID-19 pandemic reported in literature. Exposed individuals are assumed to exhibit a 5-day latency period $(\kappa=1 / 5)$, during which they can transmit the disease with a reduced infectiousness of $\rho=0.25^{32}$. Infected individuals recover and cannot infect others on average after 9 days $(\gamma=1 / 9)$ of symptom onset ${ }^{33}$. For our baseline parameters we assume $50 \%(\sigma=0.5)$ of the infections become asymptomatic ${ }^{28,29}$, with relative infectiousness of $\varepsilon=0.4^{34}$. These baseline parameters with a per-contact likelihood of infection $\beta=0.01324$ generate a basic reproductive number of $2.4^{35,36}$. The set of parameters used in our numerical experiments, unless otherwise indicated, is collected in Table 1.

\begin{tabular}{|c|l|c|c|}
\hline Par. & Description & Value & Ref \\
\hline$v_{1}\left(v_{2}\right)$ & Risk-takers (risk-evaders) utility function shape parameter & $0.1(0.05)$ & 16 \\
$\tau_{1}\left(\tau_{2}\right)$ & Risk-takers (risk-evaders) planning horizon & $14(14)$ & Assumed \\
$\delta$ & Discount factor & 0.99986 & 16 \\
$b$ & Maximum number of contacts per day & 48 & 16 \\
\hline$\beta$ & Likelihood of infection & 0.0148 & $16,35,36$ \\
$\kappa$ & Latency rate & $1 / 5$ & 32 \\
$\gamma$ & Recovery rate & $1 / 9$ & 33 \\
$\rho$ & Exposed ind. infectiousness & 0.25 & Assumed \\
$\alpha$ & Asymptomatic ind. infectiousness & 0.4 & Assumed \\
$\varepsilon$ & Reduced relative infectiousness & 0.7 & 34 \\
$\sigma$ & Proportion of asymptomatic ind. & 0.5 & 28,29 \\
$p$ & Proportion of risk-taker individuals & Variable & Assumed \\
\hline
\end{tabular}

Table 1. Baseline parameters for the constant contacts and adaptive behavior model. 


\section{Heterogeneous adaptive responses may increase the attack rate}

In this section we show there is a trade-off between the reduced infection risk by individuals adopting precautionary measures, i.e., the risk-evaders (RE, denoted by $S_{2}, E_{2}$ and $A_{2}$ ), and the non-compliant individuals, whom we refer to as the risk-takers (RT, denoted by $S_{1}, E_{1}$ and $A_{1}$ ). Moreover, we show that this trade-off is dependent on the proportion of asymptomatic cases. In Figure 1 we show selected simulations of the disease dynamics for the constant contacts model (dashed), and for the adaptive behavior model (solid), assuming a scenario where RE reduce their infection risk by $30 \%(\varepsilon=0.7)$. In panel (A) we assume $33 \%$ of the population are RT ( $p=0.33$ ), while in panel (B) we assume $66 \%$ of the population are RT $(p=0.66)$. As expected, our simulations in panel (A) show that the epidemic is mainly driven by infections in the RE population. Interestingly, despite the high prevalence levels in the RE population, the behavioral responses of the RT and the RE populations produced in this scenario are similar. Both populations reduce their respective contact rates, $C_{t}^{S_{1}}$ and $C_{t}^{S_{2}}$, by around $20 \%$ during the peak time. On the other hand, panel (B) shows a faster and earlier epidemic with a higher peak, mainly driven by infections in the RT population. In this scenario, the behavioral responses produced diverge significantly. While RT individuals reduce their contacts by $20 \%$ during the peak time (similar to the scenario in panel (A)), RE individuals reduce their contacts by around $40 \%$ during the peak time.

The scenarios chosen assume highly distinct population structures in terms of the risk-taker and risk-evader populations ( $p=0.33$ and $p=0.66$ ), which in turn produce contrasting disease dynamics (propagation speed and peak size), ultimately inducing different behavioral responses among the $S_{1}$ and $S_{2}$ populations.

-..- Constant contacts

A) $p=0.33 \quad$ - Adaptive behavior

B) $p=0.66$

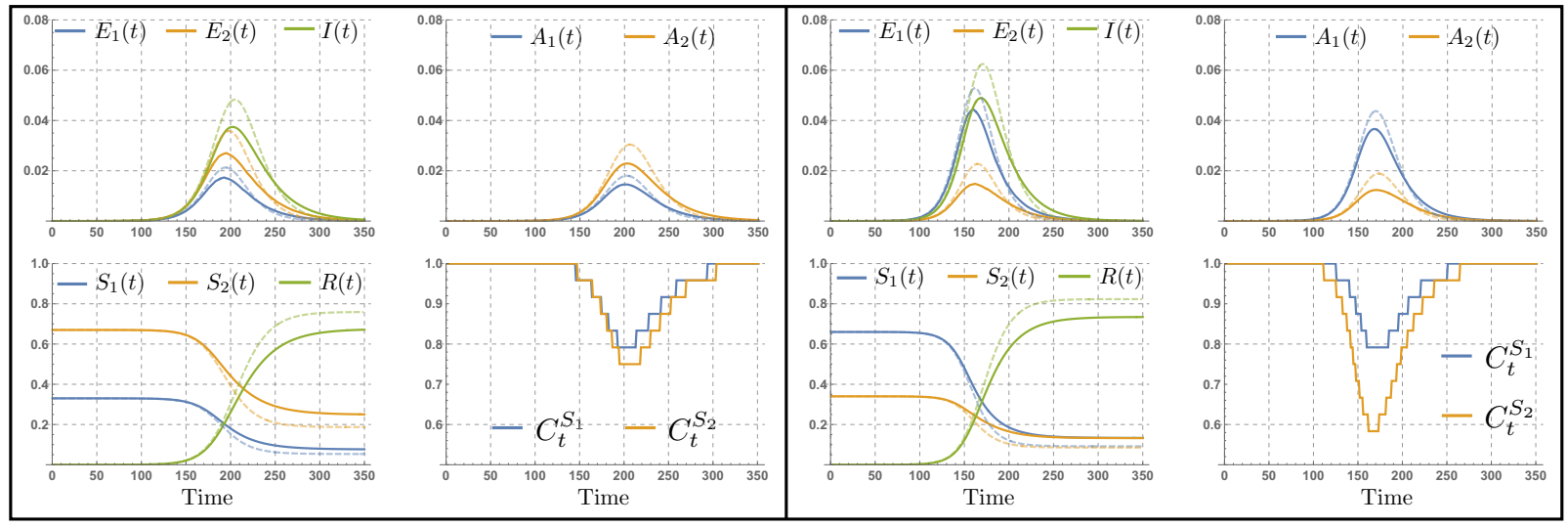

Figure 1. Variations in the proportion of RT individuals impact disease dynamics and behavioral responses. Disease dynamics under constant contacts model (dashed curves) and under adaptive behavior model (thick curves). The scenarios where 33\% (panel A) and 66\% (panel B) of the population are high-risk takers show distinct disease dynamics, inducing differential behavioral responses $\left(C_{t}^{S_{1}}\right.$ and $\left.C_{t}^{S_{2}}\right)$ as a function of the risk perception. The set of parameters used are those in Table 1.

Figure 1 show that while both scenarios exhibit reduction of contacts due to behavioral adaptations to avoid infection risk, the level of contact reduction is sensitive to the population's composition, since this impacts the prevalence levels attained. Moreover, our simulations suggest that the higher the prevalence levels reached—given by predominance of the risk-taker population - the stronger the behavioral response of the risk-evader population.

Intuitively, the extent to which efforts from the risk-evaders effectively reduce the epidemic burden depends on the proportion of risk-takers in the population. We now explore the trade-off between the risk reduction by RE individuals $(\varepsilon)$, and the proportion of risk-takers $(p)$. Notice that this trade-off is shown only on the attack rate produced for the behavioral response model. Figure 2 shows that, for the constant contacts model, the attack rate is always below the baseline scenario $(p=0, \varepsilon=1)$, monotonically decreasing as either $\varepsilon$ or $p$ decreases. This is an expected result, since the homogeneous assumption of the constant contacts model results in similarly weighting the potential risk reduction by RE individuals, and the increased infection risk that RT individuals pose to others.

However, by modeling the group-specific heterogeneous behavioral responses, we capture novel insights on the impact of RT's infectiousness and adaptive contact rates. For the adaptive behavior model, the attack rate attained in the presence of risk-takers may overcome the reduction of cases due to the RE individuals efforts, which ultimately takes the attack rate above the baseline scenario. In other words, there is trade-off between the increased expected activity and the infection risk of 

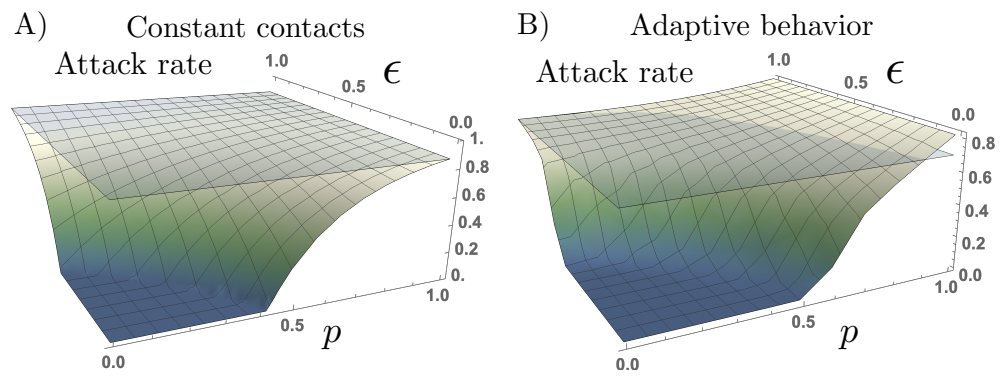

Figure 2. Risk-takers may increase the attack rate under adaptive behavioral response with uncertain information. Attack rate as a function of the proportion of both high-risk takers $(p)$ and reduced risk of infection of the risk-evaders subpopulation $(\varepsilon)$. The set of parameters used are those in Table 1.

RT individuals that balance the reduction of cases due to RE efforts. Particularly, the trade-off shown on the attack rate is a function of the proportion of RT in the population $(p)$, and the RE infection risk reduction $(\varepsilon)$.

\section{Risk misperception modulates the trade-off between risk-takers and risk-evaders}

The role of asymptomatic individuals on the spread of COVID-19 has been mostly studied from the perspective of the silent infections produced ${ }^{25-27}$. Recently, Espinoza et al. studied the potential impact that behavioral responses based on infection risk misperceptions posed by asymptomatic individuals produce on the epidemic burden ${ }^{19}$. Here, we focus on the role of asymptomatic cases on modulating the trade-off produced by the RE and RT populations. Particularly, we focus on how the presence of asymptomatic cases leading to risk misperceptions exacerbate the impact of RT individuals.

Given that the asymptomatic/symptomatic ratio, and the potential infectiousness of COVID-19 asymptomatic individuals is uncertain, we explore the impact that the proportion of asymptomatic cases has on the trade-off between the RE infection risk reduction and the proportion of RT in the population. Figure 3 exhibits the attack rates obtained by assuming different proportions of asymptomatic cases $(\sigma=0.25,0.5$ and $\sigma=0.75)$, for the different scenarios of RE efforts $(\varepsilon)$, and for varying proportions of RT individuals $(p)$.

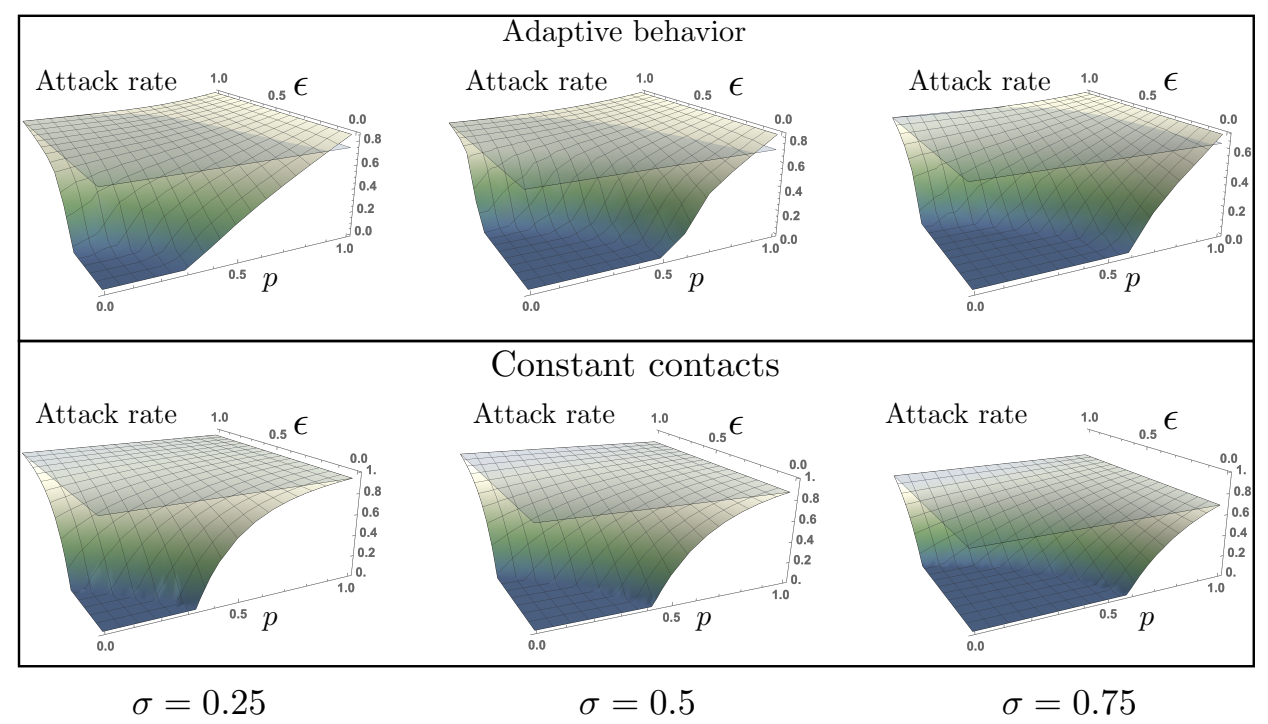

Figure 3. The trade-off between RE's risk-reduction and the proportion of RT individuals depends on the proportion of asymptomatic cases. Attack rates obtained with the constant contacts model, and with the adaptive behavior model, for the scenarios where $25 \%, 50 \%$, or $75 \%$ of infections are asymptomatic. The $(p, \varepsilon)$ regions where the attack rate is increased compared to the baseline scenario $(p=0, \varepsilon=1)$ is modulated by the presence of asymptomatic cases. The set of parameters used are those in Table 1.

We focus on the impact that varying the proportion of asymptomatic cases has on the $(p, \varepsilon)$ trade-off exhibited in the attack rate. In general, the lower the proportion of asymptomatic cases, the higher the attack levels attained. However, the lower the 
proportion of asymptomatic cases, the lower the proportion of RT individuals required to take the attack rate above the baseline level.

Our results highlight the importance of addressing differential behavioral responses during an epidemic. We show that in a population composed of two risk-groups, the consequences of differential adaptive behavioral responses can be characterized by the trade-off between the efforts to reduce infection risk and the proportion of the population not following precautionary behaviors. Moreover, our results highlight that risk-taker individuals play a dual role in the epidemic burden: besides the higher risk of infection these individuals pose to others, the high contact rates associated to this population may produce more cases than that expected using the analogous constant contacts model.

\section{Disease reporting drives behavioral responses strengths}

We study the impact that differential testing capacity has on the perceived infection risk and on the behavioral responses produced. Similar to other epidemic models including human behavioral responses ${ }^{3,37}$, our previous simulations assume a framework of complete information, however this may not be achievable for large epidemics. In reality, the risk perceptions during an epidemic, and consequently the induced behavioral responses, depend upon the region-specific surveillance efforts. We explore the impact of distinct surveillance regimes by varying the level of disease prevalence observed through testing.

We assume reporting impacts the perceived risk due to the infectious subpopulations (exposed, symptomatic and asymptomatic), proportional to its size. Figure 4 shows the impact of different levels of reporting on the contact structures of the risk-takers and risk-evaders. Our simulations show that behavioral responses of both risk-groups are highly sensitive to changes in reporting levels. Moreover, risk-evaders' behavioral responses dramatically reduce as the level of reporting decreases.
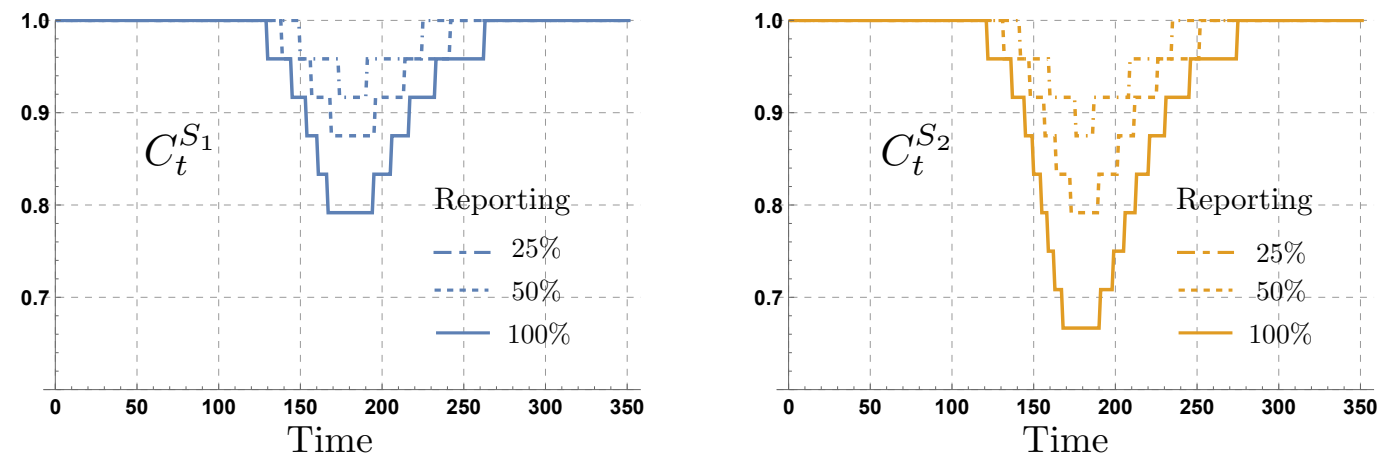

Figure 4. Risk-group contact rates for different reporting levels. Risk-taker and risk-evader contact rates for levels of reporting of $25 \%, 50 \%$ and $100 \%$. The risk-evaders' contact structure is more sensitive to changes in the reporting levels, compared to risk-takers' contact structure. The set of parameters used are those in Table 1.

Our next simulations explore the effects of different reporting levels $(\varphi)$ on the attack rate attained for the adaptive behavior model, for populations composed of different proportions of risk-takers $(p)$. Figure 5 shows that for the adaptive behavior model, the impact of risk-takers on the attack rate depends upon the reporting levels. Particularly, the extent to which behavioral responses decrease the attack rate depends on the reporting levels. For high reporting levels $(\varphi \approx 1)$, the attack rate shows a concave up shape to increments in the proportion of risk-takers. In counterpart, for low reporting levels, the attack rate shows a linear and concave down shape to increments in the proportion of risk-takers. 

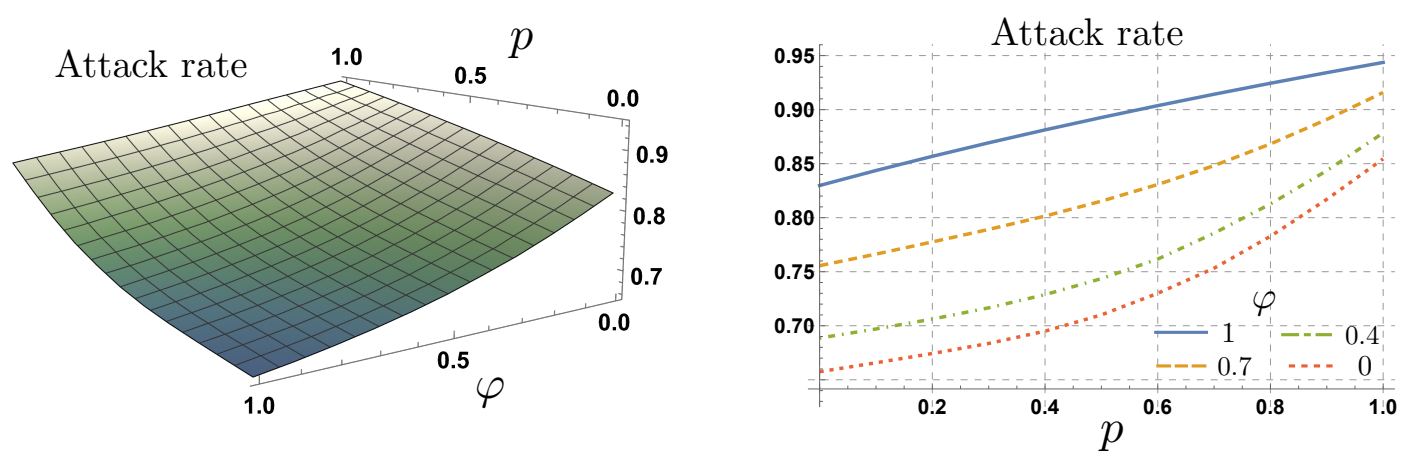

Figure 5. Attack rate for different reporting levels. Panel A shows the attack rate as a function of the reporting level $(\varphi)$, and the proportion of the population behaving as risk-takers $(p)$. Panel B shows the non-linear effect of increasing the proportion of risk takers in the population, for different values of reporting. The set of parameters used are those in Table 1.

We assume that reporting impacts all the infectious populations proportional to their sizes. In reality, reporting is mainly biased towards identifying symptomatic individuals. However, our assumption helps us to get insight on the weakening effect of under-reporting on the adaptive behavioral responses based on risk perception. Given that only a fraction of the epidemic is perceived through testing, the impact of risk misperception modulating individual behavioral responses increases as reporting decreases. In this scenario, risk misperception not only arises due to pre-symptomatic and asymptomatic individuals, but also due to the region-specific testing limitations, consequently producing a weak behavioral response in regions with very limited testing rates, even if the population is mainly composed of risk-evaders.

\section{Optimal planning horizons minimizing the attack rate}

While the heterogeneous living conditions can modulate behavioral choices leading individuals to adopt high- or low-risk behaviors, these also may impose limitations on the way individuals plan ahead and seek for the optimal future behavior. Our model of behavioral responses assumes each risk-group seeks for the contact rates that maximize their expected utility over independent planning horizons. While both optimization processes are intrinsically connected, we let each risk-group assess the potential future outcomes based on its own planning horizon. In this section we explore the impact that the planning horizons of the two risk groups, $\tau_{1}$ and $\tau_{2}$, have on the attack rate.

In Figure 6, we show selected scenarios of the attack rate for the adaptive behavior model, as a function of the RT planning horizon $\left(\tau_{1}\right)$, and the RE planning horizon $\left(\tau_{2}\right)$. We consider three scenarios: where the population is mostly composed by $\operatorname{RE}(p=0.33)$, where the population is balanced with both risk-groups $(p=0.5)$, and where most of the population are RT $(p=0.75)$. Our simulations show that depending on the population structure in terms of the RE/RT, there exists a combination of planning horizons that minimizes the attack rate. Moreover, the selected simulations show that the impact of varying the RT and the RE planning horizons is not symmetric, regardless of the RT/RE proportions. This is an expected result, given the different behavioral responses across risk-groups due to distinct infection risk perceptions.
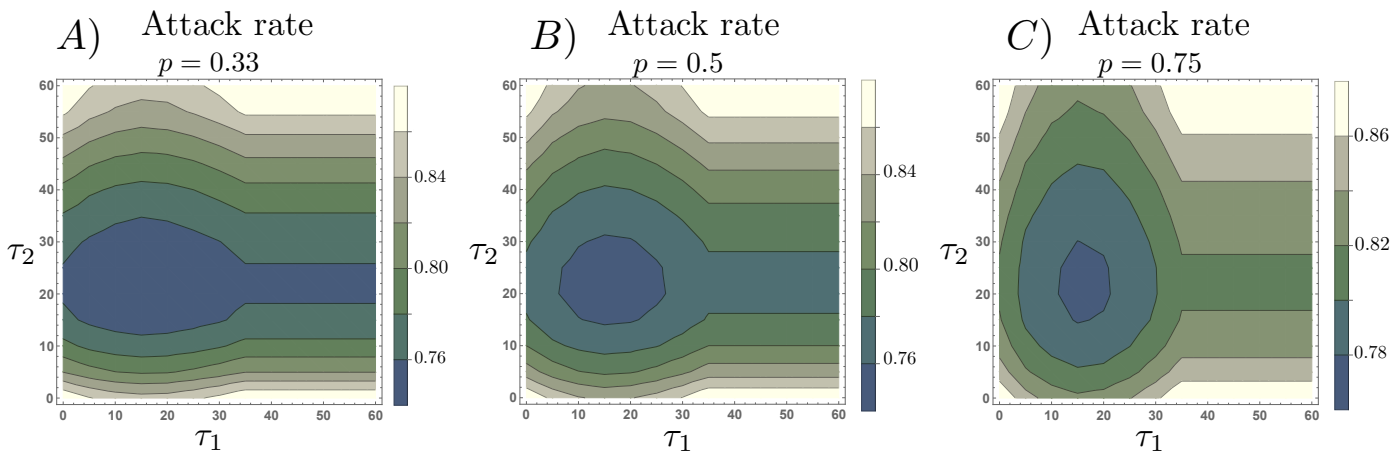

Figure 6. There is a set of planning horizons minimizing the attack rate. The figure shows attack rate level curves as a function of the risk-takers and risk-evaders planning horizons, $\tau_{1}$ and $\tau_{2}$, respectively. Differences in group-specific infection risks ( $\beta$ and $\varepsilon \beta$ ) and group-specific risk assessment modify the optimal planning horizon. The set of parameters used are those in Table 1. 
Moreover, consistent with our simulations in Figure 1, the attack rate under adaptive behavioral responses is more sensitive to changes in the planning horizon of RE individuals than for RT ones. In other words, unless the population is mainly composed of RT individuals $(p=0.75)$, increments in the planning horizon of the RE individuals $\left(\tau_{2}\right)$ impact the attack rate more than the corresponding increment of the RT population's planning horizon $\left(\tau_{1}\right)$.

\section{Discussion}

We focus on a behavioral aspect extensively documented during the COVID-19 pandemic, the population's heterogeneous behavioral responses. While the effectiveness of non-pharmaceutical interventions (NPIs) has been thoroughly studied ${ }^{12,38}$, behavioral polarization and heterogeneous adherence to recommended policies have been widely reported ${ }^{39,40}$. Political or ideological reasons, economic stress, or the lack of reasonable alternatives are aspects leading people to maintain social interactions despite the risk of COVID-19 infection ${ }^{41,42}$. The evidence to date shows that behavioral polarization is pervasive worldwide. The multiple aspects reducing NPIs compliance seem to be reinforced in scenarios where mandates rely on a decentralized governance system. For instance, before vaccines were widely available in the U.S., mask mandates were independently and unevenly enforced at different levels across counties ${ }^{43}$. Despite public health experts endorsed the effectiveness of mask wearing in multiple times, mask wearing mandates along with the effectiveness of masks were the center of national debate ${ }^{44}$. Another example is vaccine hesitancy, which has a long history of being an enormous challenge to control diseases worldwide ${ }^{45}$. Even though multiple vaccines against SARS-CoV-2 are currently available, mostly in developed countries, vaccine hesitancy continues to be a challenge in the containment of the ongoing COVID-19 pandemic ${ }^{46}$. Compliance to social distancing mandates has also shown polarized responses. Multiple social aspects like economic stress, epidemic politicization, or lack of trust in public health authorities have been reported as factors that reduce social distancing adherence ${ }^{47}$.

To reveal the importance of heterogeneous behavioral responses, we study the co-evolving dynamics of heterogeneous adaptive human behavior and disease transmission. We find that adaptive behavioral responses produced by the perceived risk of infection dynamically modify the group-specific contact structure, which modulates the epidemic dynamics. Our results exhibit a trade-off between the efforts to avoid infection by the risk-evader population, and the proportion of risk-taker individuals with relaxed infection risk perceptions. This trade-off defines a threshold in terms of the final epidemic size. Heterogeneous behavioral responses in a structured population may increase or reduce the final epidemic size, relative to final size attained by the analogous population exhibiting homogeneous behavioral responses. In other words, in a structured population, the privately optimal behavioral responses may lead to an increase in the final size of the epidemic.

On the other hand, our simulations show potential impacts of biased adaptive behavioral responses, for instance, due to uncertainty about the true health status of individuals. Given that most social interactions are subject to immediate evaluation of the infection risks, individuals respond to easily observable cues-specifically the presence or the absence of symptoms ${ }^{48}$. It follows that understanding of infection risk is assumed to be determined by vulnerability cues ${ }^{31}$ and the absence of these signals would lead to, at best, a weak behavioral response to individuals exhibiting mild or no symptoms. The impact of risk misperception on the contact structure due to the perceived lack of symptoms in the asymptomatic population has the potential to balance or be surpassed by the increasing contact rates of asymptomatic individuals, producing more secondary infections. Our simulations show that group-specific optimal behavioral responses and planning horizons may differ among individuals, depending on the associated risk of infection. We found that risk assessment, information accuracy, and willingness to follow precautionary behaviors markedly impact epidemic outcomes. We found the set of optimal planning horizons to be dependent not only on the risk perceptions of individuals across groups, but also on the population structure in terms of risk-takers and risk-evaders individuals.

We let differential risk perceptions induce heterogeneous adaptive behavioral responses among risk-taker and risk-evader individuals. To model the independently chosen but interconnected behavioral responses, our model of adaptive behavior couples a set of Markov decision processes, formalized via Bellman's equations. Our model of adaptive behavioral response is based on a projection of the system's future state up to a specific planning horizon. Individuals seek to balance the cost and benefits of social interactions over the planning horizon, subject to their group-specific risk perception. To model individual adaptive behavior during an ongoing epidemic, we use a decision-making framework that incorporates the private benefits and costs of social interactions. Individuals are assumed to be naïve to the impact their decisions impose on others, they do not realize the external costs and benefits of their behavior. Consequently, the role of empathy or social group affinities are not incorporated in the decision-making process. Another critical aspect of the behavioral model is the symmetric, uni-dimensional and single peaked utility function, widely used in economic theory ${ }^{49}$, which allows us to focus on the costs and benefits of social interaction decisions while defining a single preferred contact rate. We model optimal decision-making solely as a function of infection risk aversion. We recognize that many other factors influence the decision-making process in the ongoing pandemic, where these may be characterized as the balance of independent risks, accumulated knowledge and projected benefits ${ }^{11,50}$. Politicization of the epidemic and the limited capacity to respond by those with low incomes is partially reflected in the population's structured behavioral responses. Nonetheless, we aim to get insights on the impact that heterogeneous 
adaptive human behavior produces on the system dynamics by using a parsimonious mechanistic model. Thus, effective control measures intended to induce behavioral responses should address the potential heterogeneous compliance levels and their implications. For instance, the trade-off between reducing risk efforts and the proportion of the population following risky behaviors may be adjusted by enforcing individual-level incentives.

\section{Methods}

We study the impact of interactions between infected individuals and susceptible individuals dynamically adapting their behavior in response to the perceived risk of infection. Our model focuses on infected individuals with mild or no symptoms and excludes individuals with severe symptoms, since these have minimal interaction with the general population. The economicepidemiological model we use incorporates the interdependence between the epidemic burden and individual behavioral responses. Figure 7 shows a schematic of the coupling between the mean field epidemiological model and the Markov Decision Processes we use to model heterogeneous adaptive behavioral responses. We formulate a mean-field epidemiological model incorporating explicit contact rates to evaluate the progression of the epidemic, while simultaneously using a Markov Decision Framework to model individual adaptive behavioral responses.
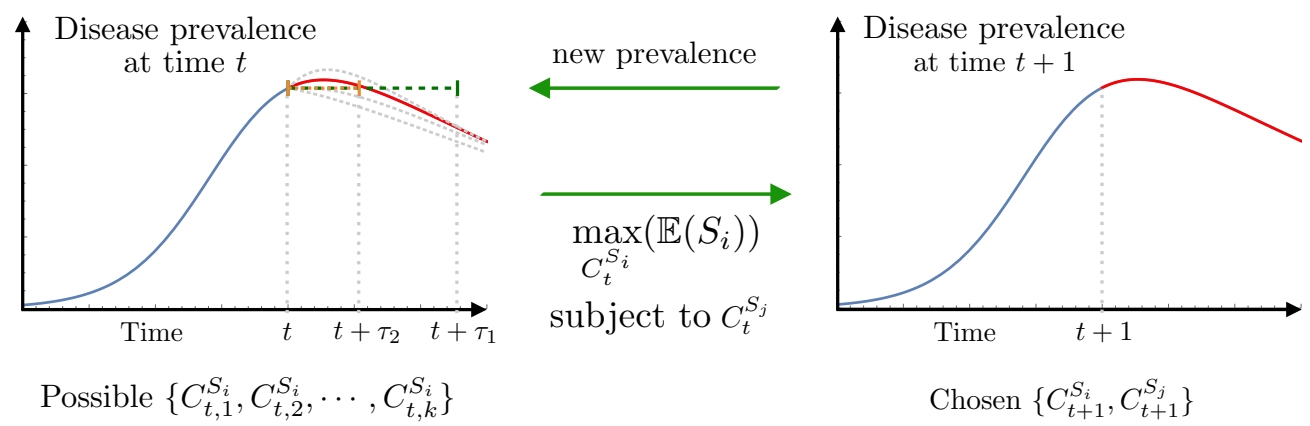

Figure 7. Coupling disease dynamics and forward looking Markov Decision Processes. At each time step the sequential decision process sets a feedback loop between the epidemic state and individual behavioral responses: $(i)$ the current disease prevalence defines potential future health state transitions, (ii) a projection of the system's future state over the planning horizon sets the optimization problem, (iii) we find the optimal contact rates and simulate the epidemic model one step forward.

Notice that the optimization processes for RT and RE susceptible individuals are intrinsically coupled. The contact rates selected by a given population affect the overall population's activity, which in turn impact the population's mixing. At each time step, we decouple these processes by computing the optimal contact rate at time $t+1$ for a given risk group, assuming the contact rate of the other risk-group to be the same as the one observed at time $t$, the latest sample available. At each time step, the group-specific optimization process incorporates a projection of the system's future state, by assuming the current prevalence remains constant over the planning horizon. Variations on the future system's state projection, and on the projection period length, deeply impact the solutions of the optimization problems, consequently impacting the outcomes of the behavioral responses and the epidemic dynamics. By solving the group-specific optimization process at each time-step, we get the privately optimal contact rate for each risk-group. This allows us to simulate the epidemic one step forward and to find the next step disease prevalence level. We iterate this process over the epidemic period to simulate the coevolution of the epidemic process and behavioral adaptations.

\section{Heterogeneous adaptive behavior}

The paradigm in our model of human adaptive behavior is given by the trade-off between increasing contacts and the differential risk of infection that these carry for the RT and the RE populations. Individuals in each health class seek to maximize the expected utility due to social interactions, while trying to minimize infection risk, according to the transmission dynamics determined by the constant contact model in the SI appendix.

Aside from the conditions that motivate heterogeneous behaviors, in our model individual behavior differs across health classes and risk groups, but individuals with similar health status and within the same risk group are assumed to behave similarly. We may expect some individuals to be satisfied with lower social activity than others however, for simplicity, we assume individual contact preferences have homogeneous and time-invariant structure. That is, individuals similarly value contacts over time, and independently of the epidemic state. Moreover, individual decentralized decisions are assumed to be taken from privately optimal perspectives. The cost-benefit trade-off perceived by individuals does not consider the aggregate effects that changing the pool of contacts has on others' decisions. 
We incorporate heterogeneous behavior in the constant contacts model by weighting the population in each health-class with the corresponding risk-group specific contact rates. Under the adaptive behavior model, the mixing is proportional to the population distribution among health-classes, and conditional on the behaviors determining the dynamic contact rates. Taking the constant contacts model in as a baseline (see SI appendix), we derive the incidence terms for the RT and RE susceptible individuals by considering the proportion of contacts that a typical individual in the $S_{1}$ and $S_{2}$ compartments makes with other infectious individuals,

$$
\beta C_{t}^{S_{1}} S_{1} \frac{\rho\left(C_{t}^{E_{1}} E_{1}+C_{t}^{E_{2}} E_{2}\right)+\alpha\left(C_{t}^{A_{1}} A_{1}+C_{t}^{A_{2}} A_{2}\right)+C_{t}^{I} I}{\sum_{h} C_{t}^{h} h}, \text { and } \varepsilon \beta C_{t}^{S_{2}} S_{2} \frac{\rho\left(C_{t}^{E_{1}} E_{1}+C_{t}^{E_{2}} E_{2}\right)+\alpha\left(C_{t}^{A_{1}} A_{1}+C_{t}^{A_{2}} A_{2}\right)+C_{t}^{I} I}{\sum_{h} C_{t}^{h} h},
$$

where $\sum_{h} C_{t}^{h} h$ is the total population activity, for individuals in health classes $h \in\left\{S_{1}, S_{2}, E_{1}, E_{2}, I, A_{1}, A_{2}, R\right\}$ selecting contact rates $\left\{C_{t}^{S_{1}}, C_{t}^{S_{2}}, C_{t}^{E_{1}}, C_{t}^{E_{2}}, C_{t}^{I}, C_{t}^{A_{1}}, C_{t}^{A_{2}}, C_{t}^{R}\right\}$, at time $t$.

Since we assume economic productivity depends exclusively on social interactions, individuals determine the daily optimal contact choices at each time step by maximizing their expected utility $V_{t}(h)$, depending on their current health status $h \in\left\{S_{1}, S_{2}, E_{1}, E_{2}, I, A_{1}, A_{2}, R\right\}$. The health-specific expected utilities $V_{t}(h)$ comprise the potential benefits obtained by making the optimal contact choice at each future time step during the group-specific planning horizon $\tau_{i}$. The expected utilities account for potential future transitions to other health states, weighted by the respective transition probabilities, which are given by the system's current state (the population distribution among health states and their respective contact choices). Individuals evaluate the future benefits/costs assuming the population distribution remains constant during the planning periods. Preferences are assumed single-peaked, so that individuals have a unique optimal contact rate in the absence of disease dynamics. Following the work by Morin et al. ${ }^{18}$, we assume a utility function of the particular form $u_{t}(h)=\left(b C_{t}^{h}-\left(C_{t}^{h}\right)^{2}\right)^{v}$, where $b$ is the per-day maximum number of contacts possible, $v$ is the utility function shape parameter, and $C_{t}^{h}$ is the contact rate of a typical individual with health status $h$ at time $t$. We assume individuals within the same risk-group get the same per-contact utility over time, regardless of their health status, except symptomatic individuals who get no utility during the infectious period. We use variations of the $v$ parameter to modify the marginal benefits of increasing contacts across groups. In other words, our model of heterogeneous behavior assumes individuals across groups show differential disposition to reduce their daily number of contacts. Moreover, we assume risk assessment remains constant over time, therefore the risk-group-specific utility function remains invariant over the epidemic period.

Finally, we incorporate the role of uncertain information on the decision-making process. We let the perceived health status represent a source of information uncertainty, where non-symptomatic individuals (exposed and asymptomatic), unaware of the infection risk they pose to others, may perceive themselves — and be perceived by others — as not presenting a risk of infection ${ }^{19}$.

Susceptible, exposed, and asymptomatic individual behavior: We model the susceptible individuals' daily optimal contact choice problem as a dynamic programming problem, the solution to which generates the privately optimal contact rate $^{16-19}$. Note that, regardless of the behavioral group, individual progression across health states follows a similar structure. Therefore, a single set of Bellman's equations formulates the optimization problem for both behavioral groups, where behavioral heterogeneity is captured by accordingly changing the health state transition probabilities. Formally, susceptible individuals' daily optimal contact rate solves the Bellman's equation,

$$
V_{t}\left(S_{i}\right)=\max _{C_{t}}\left\{u\left(S_{i}, C_{t}^{S_{i}}\right)+\delta\left[\left(1-P^{S_{i} E_{i}}\right) V_{t+1}\left(S_{i}\right)+P^{S_{i} E_{i}}\left(V_{t+1}\left(E_{i}\right)\right)\right]\right\}
$$

where $V_{t}\left(S_{i}\right)$ is the expected utility of risk group $i$ susceptible individuals at time $t, V_{t+1}\left(S_{i}\right)\left(V_{t+1}\left(E_{i}\right)\right)$ is the expected utility of being susceptible (exposed) at time $t+1$, and

$$
P^{S_{1} E_{1}}\left(C_{t}^{S_{1}}\right)=1-\exp \left(-\beta C_{t}^{S_{1}} \frac{\rho\left(C_{t}^{E_{1}} E_{1}+C_{t}^{E_{2}} E_{2}\right)+\alpha\left(C_{t}^{A_{1}} A_{1}+C_{t}^{A_{2}} A_{2}\right)+C_{t}^{I} I}{\sum_{h} C_{t}^{h} h}\right)
$$

is the probability of being infected at time $t$ for RT individuals. Since RT and RE individuals have similar disease progressions, Eqn. (2) also holds for RE, by adjusting the respective infection risk $(\varepsilon \beta)$ and the corresponding contact rate $\left(C_{t}^{S_{2}}\right)$, such that

$$
P^{S_{2} E_{2}}\left(C_{t}^{S_{2}}\right)=1-\exp \left(-\varepsilon \beta C_{t}^{S_{2}} \frac{\rho\left(C_{t}^{E_{1}} E_{1}+C_{t}^{E_{2}} E_{2}\right)+\alpha\left(C_{t}^{A_{1}} A_{1}+C_{t}^{A_{2}} A_{2}\right)+C_{t}^{I} I}{\sum_{h} C_{t}^{h} h}\right) .
$$

The optimization problem formalized in Eqn. (2) incorporates RT (RE) susceptible individuals' immediate utility $\left(u\left(S_{i}, C_{t}^{S_{i}}\right)\right.$ ), plus the expected future utility discounted at a rate $\delta$. The susceptible individuals' expected future utility accounts for the 
expected utility of remaining susceptible at the next time step, $V_{t+1}\left(S_{i}\right)$, with probability $1-P^{S_{i} E_{i}}$ and the expected utility of being infected $V_{t+1}\left(E_{i}\right)$ (progressing to the $E_{i}$ compartment), with probability $P^{S_{i} E_{i}}$.

Notice that the solution of the optimization problem for susceptible individuals depends upon the expected utility of exposed individuals. Similarly to equation (2), we formulate the Bellman's equation for exposed and asymptomatic individuals

$$
V_{t}\left(E_{i}\right)=u\left(E_{i}, C_{t}^{S_{i}}\right)+\delta\left[\left(1-P^{E_{i}}\right) V_{t+1}\left(E_{i}\right)+P^{E_{i}}\left(\sigma V_{t+1}\left(A_{i}\right)+(1-\sigma) V_{t+1}(I)\right)\right],
$$

where $P^{E_{i}}=1-e^{-\kappa}$ stands for the probability of progressing from the $E_{i}$ health class to either $A_{i}$ or $I$, with respective probabilities $\sigma$ and $1-\sigma$, and where the expected utility of asymptomatic individuals is given by,

$$
V_{t}\left(A_{i}\right)=u\left(A_{i}, C_{t}^{S_{i}}\right)+\delta\left[\left(1-P^{A_{i} R}\right) V_{t+1}\left(A_{i}\right)+P^{A_{i} R} V_{t+1}(R)\right],
$$

with $P^{A_{i} R}=1-e^{-\gamma}$ representing the probability of recovery.

We assume the absence of symptoms leads exposed and asymptomatic individuals to perceive themselves (and be perceived by others) as susceptible individuals, therefore becoming a source of uncertain information. This is incorporated in their corresponding immediate utilities on equations (5) and (6), as $u\left(E_{i}, C_{t}^{S_{i}}\right)$ and $u\left(A_{i}, C_{t}^{S_{i}}\right)$, where susceptible, exposed, and asymptomatic individuals within the same risk group choose their contact rates in the same way. In other words, we track individual risk-avoidance efforts over health-classes except while infected and recovered.

Symptomatic and recovered individual behavior: Since our model for disease progression does not consider potential reinfections, we assume there is no incentive for symptomatic and recovered individuals to adapt their behavior. It follows that symptomatic and recovered individuals make the daily number of contacts that maximizes their net benefits. The expected utility of symptomatic individuals, $V_{t}(I)$, is given by the Bellman's equation,

$$
V_{t}(I)=u\left(I, C_{t}^{I *}\right)+\delta\left[\left(1-P^{I R}\right) V_{t+1}(I)+P^{I R} V_{t+1}(R)\right],
$$

while the expected utility of recovered individuals, $V_{t}(R)$, is formalized by,

$$
V_{t}(R)=u\left(R, C_{t}^{R *}\right)+\delta V_{t+1}(R),
$$

where $P^{I R}=1-\exp ^{-\gamma}$ is the recovery probability.

Note that symptomatic and recovered individual utility expectations represent static problems, since they only depend upon the recovery rate and can be explicitly solved. Although we have not explicitly included a potential contact rate reduction of symptomatic individuals, for instance, due to altruism or sanctions, we can model it by limiting the maximum contacts available for this subpopulation.

\section{References}

1. Murray, D. R. \& Schaller, M. The behavioral immune system: Implications for social cognition, social interaction, and social influence. In Advances in experimental social psychology, vol. 53, 75-129 (Elsevier, 2016).

2. Schaller, M., Murray, D. R. \& Hofer, M. K. The behavioural immune system and pandemic psychology: the evolved psychology of disease-avoidance and its implications for attitudes, behaviour, and public health during epidemic outbreaks. Eur. Rev. Soc. Psychol. 1-37 (2021).

3. Funk, S., Salathé, M. \& Jansen, V. A. A. Modelling the influence of human behaviour on the spread of infectious diseases: A review. J. The Royal Soc. Interface 7, 1247-1256, DOI: 10.1098/rsif.2010.0142 (2010).

4. Verelst, F., Willem, L. \& Beutels, P. Behavioural change models for infectious disease transmission: A systematic review (2010-2015). J. The Royal Soc. Interface 13, 20160820 (2016).

5. Chen, J. et al. Individual and collective behavior in public health epidemiology. In Handbook of statistics, vol. 36, 329-365 (Elsevier, 2017).

6. Thunström, L., Newbold, S. C., Finnoff, D., Ashworth, M. \& Shogren, J. F. The benefits and costs of using social distancing to flatten the curve for covid-19. J. Benefit-Cost Analysis 11, 179-195 (2020).

7. Koren, M. \& Petô, R. Business disruptions from social distancing. Plos one 15, e0239113 (2020).

8. Fenichel, E. P. Economic considerations for social distancing and behavioral based policies during an epidemic. J. health economics 32, 440-451 (2013).

9. Moya, C. et al. Dynamics of behavior change in the covid world. Am. J. Hum. Biol. (2020). 
10. Venkatesh, A. \& Edirappuli, S. Social distancing in covid-19: what are the mental health implications? Bmj 369 (2020).

11. Pagliaro, S. et al. Trust predicts covid-19 prescribed and discretionary behavioral intentions in 23 countries. PloS one 16, e0248334 (2021).

12. Haischer, M. H. et al. Who is wearing a mask? gender-, age-, and location-related differences during the covid-19 pandemic. Plos one 15, e0240785 (2020).

13. Khubchandani, J. et al. Covid-19 vaccination hesitancy in the united states: a rapid national assessment. J. Community Heal. 46, 270-277 (2021).

14. Wilder, B. et al. Modeling between-population variation in covid-19 dynamics in hubei, lombardy, and new york city. Proc. Natl. Acad. Sci. 117, 25904-25910 (2020).

15. Brzezinski, A., Kecht, V., Van Dijcke, D. \& Wright, A. L. Science skepticism reduced compliance with covid-19 shelter-in-place policies in the united states. Nat. Hum. Behav. 1-9 (2021).

16. Fenichel, E. P. et al. Adaptive human behavior in epidemiological models. Proc. Natl. Acad. Sci. 108, 6306-6311 (2011).

17. Perrings, C. et al. Merging economics and epidemiology to improve the prediction and management of infectious disease. EcoHealth 11, 464-475 (2014).

18. Morin, B. R., Fenichel, E. P. \& Castillo-Chavez, C. SIR dynamics with economically driven contact rates. Nat. Resour. Model. 26, 505-525 (2013).

19. Espinoza, B., Marathe, M., Swarup, S. \& Thakur, M. Asymptomatic individuals can increase the final epidemic size under adaptive human behavior. Sci. reports 11, 1-12 (2021).

20. Kolobov, A. Planning with markov decision processes: An ai perspective. Synth. Lect. on Artif. Intell. Mach. Learn. 6, $1-210$ (2012).

21. Bennett, C. C. \& Hauser, K. Artificial intelligence framework for simulating clinical decision-making: A markov decision process approach. Artif. intelligence medicine 57, 9-19 (2013).

22. Zou, H., Su, H., Song, S. \& Zhu, J. Understanding human behaviors in crowds by imitating the decision-making process. In Thirty-Second AAAI Conference on Artificial Intelligence (2018).

23. Jackson, M. O., Rogers, B. W. \& Zenou, Y. The economic consequences of social-network structure. J. Econ. Lit. 55, 49-95 (2017).

24. Granovetter, M. The impact of social structure on economic outcomes. J. Econ. Perspectives 19, 33-50 (2005).

25. Moghadas, S. M. et al. The implications of silent transmission for the control of COVID-19 outbreaks. Proc. Natl. Acad. Sci. 117, 17513-17515 (2020).

26. Li, R. et al. Substantial undocumented infection facilitates the rapid dissemination of novel coronavirus (SARS-CoV-2). Science 368, 489-493 (2020).

27. Laxminarayan, R. et al. Epidemiology and transmission dynamics of COVID-19 in two Indian states. Science (2020).

28. Yanes-Lane, M. et al. Proportion of asymptomatic infection among COVID-19 positive persons and their transmission potential: A systematic review and meta-analysis. PloS ONE 15, e0241536 (2020).

29. Byambasuren, O. et al. Estimating the extent of true asymptomatic COVID-19 and its potential for community transmission: Systematic review and meta-analysis. Available at SSRN 3586675 (2020).

30. Meyerowitz, E. A., Richterman, A., Bogoch, I. I., Low, N. \& Cevik, M. Towards an accurate and systematic characterisation of persistently asymptomatic infection with SARS-CoV-2. The Lancet Infect. Dis. (2020).

31. Schaller, M. The behavioural immune system and the psychology of human sociality. Philos. Transactions Royal Soc. B: Biol. Sci. 366, 3418-3426 (2011).

32. Furukawa, N. W., Brooks, J. T. \& Sobel, J. Evidence supporting transmission of severe acute respiratory syndrome coronavirus 2 while presymptomatic or asymptomatic. Emerg. infectious diseases 26 (2020).

33. Wölfel, R. et al. Virological assessment of hospitalized patients with COVID-2019. Nature 581, 465-469 (2020).

34. Oliveira, J. F. et al. Mathematical modeling of COVID-19 in 14.8 million individuals in Bahia, Brazil. Nat. communications 12, 1-13 (2021).

35. Shi, Q. et al. Effective control of SARS-CoV-2 transmission in Wanzhou, China. Nat. Medicine 27, 86-93 (2021). 
36. Zhao, S. et al. Preliminary estimation of the basic reproduction number of novel coronavirus (2019-nCoV) in China, from 2019 to 2020: A data-driven analysis in the early phase of the outbreak. Int. J. Infect. Dis. 92, 214-217 (2020).

37. Reluga, T. C. Game theory of social distancing in response to an epidemic. PLoS computational biology 6 , e1000793 (2010).

38. Li, T., Liu, Y., Li, M., Qian, X. \& Dai, S. Y. Mask or no mask for covid-19: A public health and market study. PloS one 15, e0237691 (2020).

39. Nivette, A. et al. Non-compliance with covid-19-related public health measures among young adults in switzerland: Insights from a longitudinal cohort study. Soc. science \& medicine 268, 113370 (2021).

40. Betsch, C. et al. Social and behavioral consequences of mask policies during the covid-19 pandemic. Proc. Natl. Acad. Sci. 117, 21851-21853 (2020).

41. Santora, M. \& Kwai, I. As virus surges in europe, resistance to new restrictions also grows. New York Times Oct. 9, 2020 (2020).

42. Wong, C. M. L. \& Jensen, O. The paradox of trust: perceived risk and public compliance during the covid-19 pandemic in singapore. J. Risk Res. 23, 1021-1030 (2020).

43. Cunningham, G. B. \& Nite, C. Demographics, politics, and health factors predict mask wearing during the covid-19 pandemic: a cross-sectional study. BMC Public Heal. 21, 1-9 (2021).

44. Cerbin, L., DeJesus, J., Warnken, J. \& Gokhale, S. S. Unmasking the mask debate on social media. In 2021 IEEE 45th Annual Computers, Software, and Applications Conference (COMPSAC), 677-682 (IEEE, 2021).

45. Kestenbaum, L. A. \& Feemster, K. A. Identifying and addressing vaccine hesitancy. Pediatr. annals 44, e71-e75 (2015).

46. Machingaidze, S. \& Wiysonge, C. S. Understanding covid-19 vaccine hesitancy. Nat. Medicine 27, 1338-1339 (2021).

47. Painter, M. \& Qiu, T. Political beliefs affect compliance with covid-19 social distancing orders. Covid Econ. 4, 103-123 (2020).

48. Kramer, P. \& Bressan, P. Infection threat shapes our social instincts. Behav. Ecol. Sociobiol. 75, 1-18 (2021).

49. Coombs, C. H. \& Avrunin, G. S. Single-peaked functions and the theory of preference. Psychol. review 84, 216 (1977).

50. Fazio, R. H. et al. Who is (not) complying with the us social distancing directive and why? testing a general framework of compliance with virtual measures of social distancing. PloS one 16, e0247520 (2021).

\section{Acknowledgements}

The authors would like to thank members of the Biocomplexity COVID-19 Response Team and the Network Systems Science and Advanced Computing (NSSAC) Division for their thoughtful comments and suggestions related to epidemic modeling and response support. We thank members of the Biocomplexity Institute and Initiative, University of Virginia, for useful discussion and suggestions. This work was partially supported by National Institutes of Health (NIH) Grant 1R01GM109718, NSF BIG DATA Grant IIS-1633028, NSF Grant No.: OAC-1916805, NSF Expeditions in Computing Grant CCF-1918656, CCF-1917819, NSF RAPID CNS-2028004, NSF RAPID OAC-2027541, US Centers for Disease Control and Prevention 75D30119C05935, University of Virginia Strategic Investment Fund award number SIF160, and Defense Threat Reduction Agency (DTRA) under Contract No. HDTRA1-19-D-0007. Any opinions, findings, and conclusions or recommendations expressed in this material are those of the author(s) and do not necessarily reflect the views of the funding agencies.

\section{Competing interests:}

The authors declare that no competing interests exist. 


\section{Supplementary Files}

This is a list of supplementary files associated with this preprint. Click to download.

- AdaptivetworiskgroupsSI.pdf 